urinary tract have been reported (2). The organism enters the testis from the epididymis by direct invasion. Most patients with epididymal TB have no evidence of renal TB. Almost all patients with epididymal and testicular TB do not have urinary symptoms.

GUTB is common in the 20-50 years age group and not in the old, frail patients. Diagnosis in the initial stage of the disease is difficult. Painful enlargement of the testis and epididymis simulates bacterial orchitis. Later the swelling becomes nodular, firm and painless. Most of the time, M. tuberculosis cannot be isolated from urine. Presence of a sinus in the scrotum suggests the diagnosis. Otherwise biopsy of the epididymis or orchiectomy is necessary to confirm the diagnosis before commencing treatment. Fine needle aspiration cytology of the epididymal mass may suggest the diagnosis in some. The clinical features may simulate a testicular tumour. About $10 \%$ of patients with tuberculous orchitis may undergo inguinal (high) orchiectomy for a suspected tumour (3). Though Mantoux test is useful in the diagnosis of renal TB, it is normal in most patients with epididymal TB. Same is true for ESR.
Epididymal and testicular $\mathrm{TB}$ can be treated effectively with the standard six-month regime of anti-tubercular therapy. Few patients with a persistent scrotal sinus my require epididymectomy later. The transmission of genital TB from male to female is very rare. Occasional reports of pelvic tuberculosis in the sexual partner of patients with tuberculous epididymo-orchitis suggest the possibility of female to male transmission.

\section{References}

1. Annual report of the National Programme for Tuberculosis Control and Chest Diseases 2007 Ministry of Healthcare \& Nutrition, Sri Lanka.

2. Cek M, Lenk S, Naber KG, Bishop MC et al. EAU guidelines for the management of genitourinary tuberculosis. European Urology 2005; 48: 353-62

3. Viswaroop BS, Kekre N, Gopalakrishnan G. A study of isolated epididymal tuberculosis. Journal of Postgraduate Medicine 2005; 81: 109-11.

\title{
A girl with complicated Stevens Johnson syndrome following ciprofloxacin; a case report
}

\author{
Nanayakkara PGCJ, Lekamwasam S, Pratheepan GJ \\ University Medical Unit, Teaching Hospital, Karapitiya, Galle \\ Corresponding author; Dr.P.G.C.J.Nanayakkara (chathu.nanayakkara@yahoo.com)
}

\section{Introduction}

Stevens Johnson syndrome (SJS) is a multi-systemic disorder with predominant dermatological manifestations. We report a patient who had multiorgan involvement with life threatening complications.

\section{Case Report}

An eighteen year old, previously healthy girl presented to a General Practitioner with fever, dysuria and right sided loin pain of three days duration. After urinanalysis, she has been started on oral ciprofloxacin. About ten hours after the first dose, she noticed a painful skin rash over the body, lips and vulva and felt gritty sensation in the eyes.

On admission, she was febrile $\left(102^{\circ} \mathrm{F}\right)$. Diffuse mucosal ulcerations in conjunctiva, oral cavity and vulva were seen while vesiculo-bullous and targetoid eruptions were detected in the body and limbs. Furthermore, she was detected to have severe membranous conjunctivitis with epithelial defects and conjunctival dryness, favoring an allergic reaction. The clinical diagnosis of SJS was made and supportive care was initiated. 
The following day, she developed progressive haematuria with ultrasound evidence of bilateral hydroureteronephrosis and a bladder haematoma. Genito-urinary surgeon considered it to be a manifestation of SJS. Her renal functions were normal then.

Eleven days after admission, patient developed features of acute cholestatic hepatitis with AST; 440U/L, ALT; 960U/L, total S. Bilirubin; $247 \mu \mathrm{mol} / \mathrm{L}$

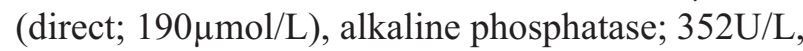
total S. protein; 86g/L (albumin; 43g/L), PT; $12 \mathrm{~s}$ (INR; 1.0). Repeat US scan showed no biliary obstruction. Serological tests for mycoplasma, EBV, CMV, HSV, hepatitis A, B or C were negative. ANA or dsDNA were not detected while ESR and CRP remained normal. The diagnosis of cholestatic hepatitis associated with SJS induced by ciprofloxacin was considered and oral prednisolone was started.

After one month of illness, she became progressively breathless with episodic wheezing. Examination revealed scattered rhonchi bilaterally and the possibility of an acute bronchitis was considered. Sputum culture and examination for AFB were negative. Her CRP remained normal during this period but serial chest radiographs revealed the presence of subcutaneous and mediastinal emphysema. The gastrograffin study preformed to exclude possibility of oesophageal rupture was normal. Arterial blood gases showed the presence of Type II respiratory failure and she was transferred for intensive care. While in ITU, she developed two brief attacks of generalized tonic clonic seizures. Biochemistry showed hyponatraemia (128 \& $124 \mathrm{mmol} / \mathrm{L})$ and hypocalcaemia $(6.4 \mathrm{mg} / \mathrm{dL})$ while EEG was normal.

The diagnosis was reviewed and further investigations were arranged to exclude other multisystemic diseases. ANA (repeated), c-ANCA, pANCA, HIV $1 \& 2$ were negative. She was started on intravenous methylprednisolone, inhaled steroids and bronchodilators. Chest HRCT revealed patches of pulmonary infiltrations in both upper lobes with widespread bronchiectatic changes (Fig 1).

She was detected to have side-effects of systemic corticosteroids over the next few weeks; marked hirsutism, proximal myopathy, monomorphic acne, persistent neutrophil leucocytosis, hypokalaemia, hypocalcaemia and acute psychosis. Alendronate
$70 \mathrm{mg} / \mathrm{wk}$ was added as she was found to have low bone mineral density (Lumbar spine Z score; -2.3).

Although patient showed a symptomatic improvement, cholestasis was progressive and liver biopsy confirmed cholestasis, probably due to large bile duct obstruction with no features of vasculitis (Fig 2). She was referred to a gastroenterologist and started on ersodeoxycholic acid. Her liver functions showed a steady improvement while on ersodeoxycholic acid but ALT, AST and alkaline phosphatase still remains elevated 8 months after her illness.

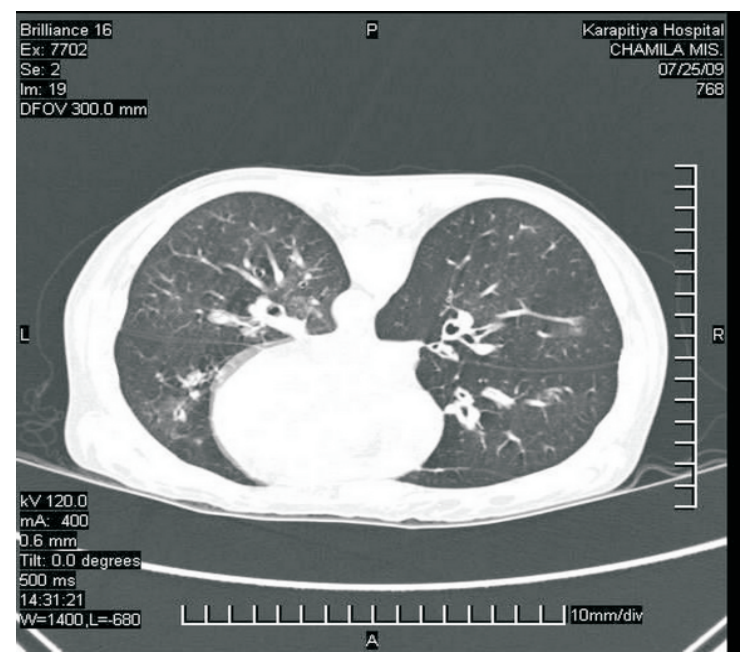

Figure 1 - Chest CT appearance

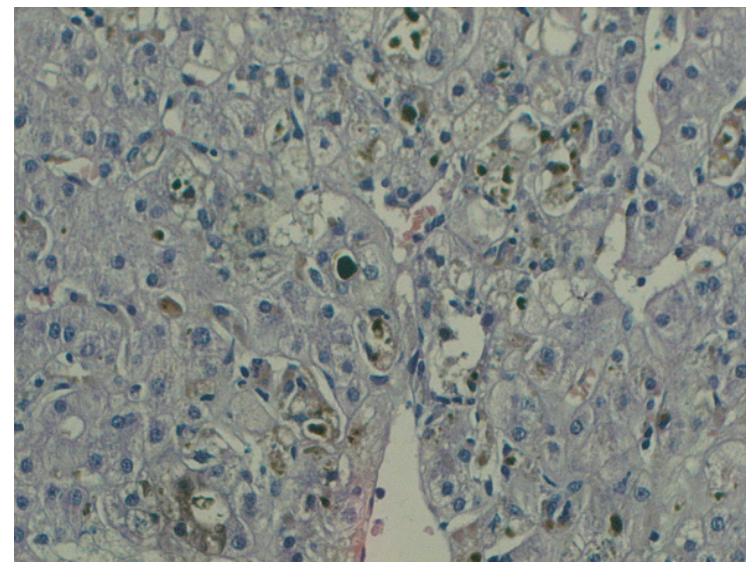

Figure 2 - Liver histology

\section{Discussion}

The exact mechanism of systemic involvement in SJS is not known. It can be a part of drug hypersensitivity $(1,2)$ affecting conjunctiva, trachea, gastrointestinal tract and genitourinary tract mainly (2). Intestinal and pulmonary involvement is 
associated with poor prognosis (1). Hepatitis is the most common manifestation while renal and respiratory dysfunction also has been common (3).

Ciprofloxacin itself is potentially hepatotoxic and this is considered to be an idiosyncratic reaction (4). Severe cases may even end up in a condition called "Vanishing bile duct syndrome"(2). Diagnostic criteria for ciprofloxacin-induced hepatitis have been documented (4).

Pulmonary complications following SJS may be progressive, ultimately requiring lung transplantation (5) or even death (6). Reported complications with SJS (and toxic epidermal necrolysis) are chronic bronchitis/bronchiolitis with obstructive changes (bronchiolitis obliterans and bronchiolitis obliterans organizing pneumonia BOOP), respiratory tract obstruction and bronchiectasis (6). There had been cases with subcutaneous and/or mediastinal emphysema as in this patient (3). About $40 \%$ of these would die while the rest would be suffering long term (6). Some have shown renal dysfunction requiring haemodialysis also (3). There are reports of encephalopathy, gastrointestinal disorders and even myocarditis as severe systemic manifestations (3).

As no specific curative treatment yet exists $(1,6,7)$, the outcome in severe cases is poor and unpredictable. The efficacy of steroids is controversial (7) and other modalities of treatment such as immunoglobulin, plasmapharesis and immunomodulaters have not shown positive results (1). Recent studies have shown a strong genetic susceptibility in drug hypersensitivities (2).

\section{References}

1. Jean- Claude Roujeau, Robert S.Stern, Bruce U. Wintroub. Cutaneous drug reactions. In: Harrison's Principles of Internal Medicine. $17^{\text {th }}$ edition 2008; pg 343- 346.

2. Gokhan Okan, Serpil Yaylaci, Onder Peker et al. Vanishing bile duct and Stevens Johnson syndrome associated with ciprofloxacin treated with tacrolimus. World Journal of Gastroenterology 2008; 14(29): 4697-700.

3. Yamiko Yamane, Michiko Aihara and Zearo Ikezana. Analysis of Stevens Johnson syndrome \& Toxic Epidermal Necrolysis in Japan from 2000 to 2006. Allergology International 2007; 56: 419-25.

4. Kapil M. Bhagirath. A case report of highly suspected ciprofloxacin induced hepatotoxicity. The Turkish Journal of Gastroenterology 2008, 19(3): 204-6.

5. Moonnumakal SP, Fan LL. Bronchiolitis obliterans in children. Curr Opin Pediatr 2008; 20(3): 272-8.

6. Kamada N, Kinoshita K, Togara Y et. al. Chronic pulmonary complications associated with Toxic Epidermal Necrolysis: report of a severe case with anti- Ro/SS- A \& a review of published work. J Dermatol 2006; 33(9): 616-22.

7. Sane SP, Bhatt AD. Stevens Johnson syndrome \& Toxic Epidermal Necrolysis - Challenges of recognition and management. J Assoc. Physicians India 2000; 48(10): 9991003. 